\title{
November 2004 space weather events: Real-time observations and forecasts
}

\author{
L. Trichtchenko, ${ }^{1}$ A. Zhukov, ${ }^{2}$ R. van der Linden, ${ }^{2}$ S. M. Stankov, ${ }^{3}$ N. Jakowski, ${ }^{3}$ \\ I. Stanisławska, ${ }^{4}$ G. Juchnikowski, ${ }^{4}$ P. Wilkinson, ${ }^{5}$ G. Patterson, ${ }^{5}$ \\ and A. W. P. Thomson ${ }^{6}$ \\ Received 11 September 2006; revised 19 January 2007; accepted 25 January 2007; published 7 June 2007.
}

[1] Space weather events with their solar origin and their distribution through the heliosphere affect the whole magnetosphere-ionosphere-Earth system. Their real-time monitoring and forecasting are important for science and technology. Here we discuss one of the largest space weather events of Solar Cycle 23, in November 2004, which was also one of the most difficult periods to forecast. Nine halo coronal mass ejections (CMEs), interacting on their way through the interplanetary medium and forming two geoeffective interplanetary structures, exemplify the complexity of the event. Real-time and quasi-real-time observations of the ground geomagnetic field show rapid and extensive expansion of the auroral oval to $55^{\circ}$ in geomagnetic latitude accompanied by great variability of the ionosphere.

Geomagnetically induced currents (GICs) seen in ground networks, such as power grids and pipelines, were significant during the event, although no problems were reported. Forecasts of the CME propagation, global and local ground geomagnetic activity, and ionospheric parameters, issued by several regional warning centers, revealed certain deficiencies in predictions of the interplanetary characteristics of the $\mathrm{CME}$, size of the geomagnetic disturbances, and complexity of the ionospheric variations produced by this event. This paper is a collective report based on the materials presented at the splinter session on November 2004 events during the first European Space Weather Week.

Citation: Trichtchenko, L., A. Zhukov, R. van der Linden, S. M. Stankov, N. Jakowski, I. Stanisławska, G. Juchnikowski, P. Wilkinson, G. Patterson, and A. W. P. Thomson (2007), November 2004 space weather events: Real-time observations and forecasts, Space Weather, 5, S06001, doi:10.1029/2006SW000281.

\section{Introduction}

[2] The declining phase of Solar Cycle 23 was lit up by a burst of solar activity at the beginning of November 2004. This resulted in a cluster of disturbances propagating through the interplanetary medium and producing two large geomagnetic storms with maximum $K p$ index of 9 - and Dst of -373. This paper provides a detailed description of the solar-magnetosphere-ionosphere conditions for the period of 6-10 November using real-time and quasireal-time data (sections $2-4$ ). These observations are avail-

\footnotetext{
${ }^{1}$ Geomagnetic Laboratory, Natural Resources Canada, Ottawa, Ontario, Canada.

${ }^{2}$ Solar Influences Data Analysis Center, Royal Observatory, Brussels, Belgium.

${ }^{3}$ Institute of Communications and Navigation, German Aerospace Centre, Neustrelitz, Germany.

${ }^{4}$ Space Research Centre, Warsaw, Poland.

${ }^{5}$ Ionospheric Prediction Service Radio and Space Services, Haymarket, New South Wales, Australia.

${ }^{6}$ British Geological Survey, Edinburgh, UK.
}

able on line from a number of different types of sources, such as SOHO, ACE, GOES, and GPS satellites, as well as from the ground-based monitoring of the geomagnetic field (INTERMAGNET) and ionosphere (GNSS, ionosondes). The paper also includes observations provided by engineers and researchers dealing with ground infrastructures affected by space weather, such as power systems and pipelines (section 5). Finally, forecasts, issued by the International Space Environment Service's regional warning centers (RWCs) are discussed in section 6 .

[3] A recent example of the extensive retrospective space weather case study is the 72 papers on the OctoberNovember 2003 storms, published in the three-journal special section "Violent Sun-Earth Connection Events of October-November 2003" in Journal of Geophysical Research, 110(A9), 2005; Geophysical Research Letters, 32(3, 12), 2005; and Space Weather, 2, 2004 and 3, 2005. This paper reports on the November 2004 space weather event as it was seen during its progression and is based mostly on the data available at the time of the event. Focusing on 
Table 1. List of Halo CMEs Produced by NOAA AR 10696 in November 2004

\begin{tabular}{cccccc}
\hline CME & Date & $\begin{array}{c}\text { CME Time of } \\
\text { First Appearance in } \\
\text { LASCO C2 }\end{array}$ & $\begin{array}{c}\text { FOV Speed, } \\
\mathrm{km} / \mathrm{s}\end{array}$ & $\begin{array}{c}\text { Peak of } \\
\text { Accompanying Flare }\end{array}$ & Location \\
\hline 1 & 3 Nov & 0354 UT, partial halo & 750 & M1.6, 0335 & N09E45 \\
2 & 3 Nov & 1606 UT, full halo & 1016 & M5.0, 1547 & N11E40 \\
3 & 4 Nov & 09:4 UT, partial halo & 635 & C6.3, 0905 & N08E28 \\
4 & 4 Nov & 2330 UT, partial halo & 1053 & M2.5, 2229 & N11E19 \\
& & & & M5.4, 2309 & N11E19 \\
5 & 6 Nov & 0131 UT, partial halo & 960 & M9.3, 0034 & N10E05 \\
& & & & M3.9, 0057 & N07E00 \\
6 & & & 1770 & X2.0, 1606 & N09W17 \\
7 & 7 Nov & 1706 UT, full halo & 520 & C7.9, 0329 & N08W28 \\
8 & 8 Nov & 0406 UT, full halo & 1853 & M8.9, 1719 & N07W51 \\
9 & 9 Nov & 1726 UT full halo & 1975 & X2.5, 0213 & N09W49 \\
\hline
\end{tabular}

the needs of the specific users of real-time data and forecasts, the description of the event gives a more detailed view of the local effects, such as instantaneous geomagnetic field data or maps of ionospheric parameters for specific latitudinal and longitudinal zones, rather than global geomagnetic indices and median ionospheric parameters (which are also presented in the paper).

[4] We also address the problems and difficulties of forecasting the geoeffectiveness of the November 2004 events, specifically, time, amplitude, and duration of their effects on the Earth's geomagnetic field and ionosphere. Unlike the October 28-31 2003 events, where Earthdirected halo coronal mass ejections (CMEs) on 28 and 29 October produced well-separated interplanetary structures resulting in well-defined periods of geomagnetic storms on 28-29 and 31 October 2003 [Gopalswamy et al., 2005, Figure 1], the two large jumps in geomagnetic activity in November 2004 were presumably caused by multiple interacting CMEs making it difficult to forecast or even to determine the direct connection between an individual CME and its geophysical effect, if any.

[5] Over time, the different RWCs have developed forecasts for different types of applications, such as for geomagnetically induced currents (GICs) in power systems and pipelines or for the conditions of HF propagation. Although some attempts to compare the prediction performances of the different centers have been made [Oler, 2004], we found that the current wide variety of forecasts made comparisons unhelpful, especially in the case of the complex event of November 2004. Hence in this paper we feel it is more effective to describe the forecasts produced by each RWC and problems forecasters experienced during the event and to identify some ways these forecasts could be improved.

\section{Solar Sources and Associated Interplanetary Disturbances}

[6] Solar observations are provided by a number of ground-based observatories around the globe, together with space-based observations from the Solar and Heliospheric Observatory (SOHO), Geostationary Operational
Environmental Satellites (GOES), and Advanced Composition Explorer (ACE) satellites. SOHO observations are especially important for forecasting the propagation of a solar disturbance to the Earth. This section describes the solar and interplanetary situation that later leads to the effects on the Earth's magnetosphere and ionosphere.

[7] In November 2004, ground solar observatories noted active region (AR) 10696 as it appeared on the east limb. The region grew rapidly from 21 millionths of the solar hemisphere on 1 November to its peak of 910 millionths by 6 November. During 2-7 November, the GOES X-ray sensor detected eleven $M$ flares and two $X$ flares produced by this region. Most of these strong flares were accompanied by full or partial halo CMEs observed by $\mathrm{SOHO} /$ LASCO, some of them with a complex configuration due to the probable interaction of two or more events (e.g., early on 6 November). All the CMEs had clear low corona signatures (coronal dimming and extreme ultraviolet imaging telescope (EIT) waves) detected by SOHO/EIT; hence the source region of the CMEs was unambiguously identified to be in or near AR 10696.

[8] Table 1 lists each CME with accompanying flares and their locations on the solar disk for the period 3-10 November 2004. While the solar sources of the November space weather events were relatively clear (with CMEs 4, 5 , and 6 possibly produced by two or more eruptions), their subsequent interplanetary structures became complicated after the CME left the Sun. Real-time plots of the solar wind characteristics observed at the ACE satellite are shown in Figures $1 \mathrm{a}-1 \mathrm{e}$, with GOES 5-min proton flux shown in Figure 1f.

[9] The tentative scenario for the propagation of nine halo CMEs and identification of the interplanetary structures, which was typical of forecaster thinking during the progress of the disturbances, can be described as follows. The first CME probably missed the Earth, as no distinctive signature was found in the ACE solar wind data, most likely because its origin was located too far from the central meridian. The following five halo CMEs may correspond to the five shocks, observed on 7 and 9 November. Their approximate times of occurrence are marked as blue dashed lines in Figure 1e. The slow and weak full halo 

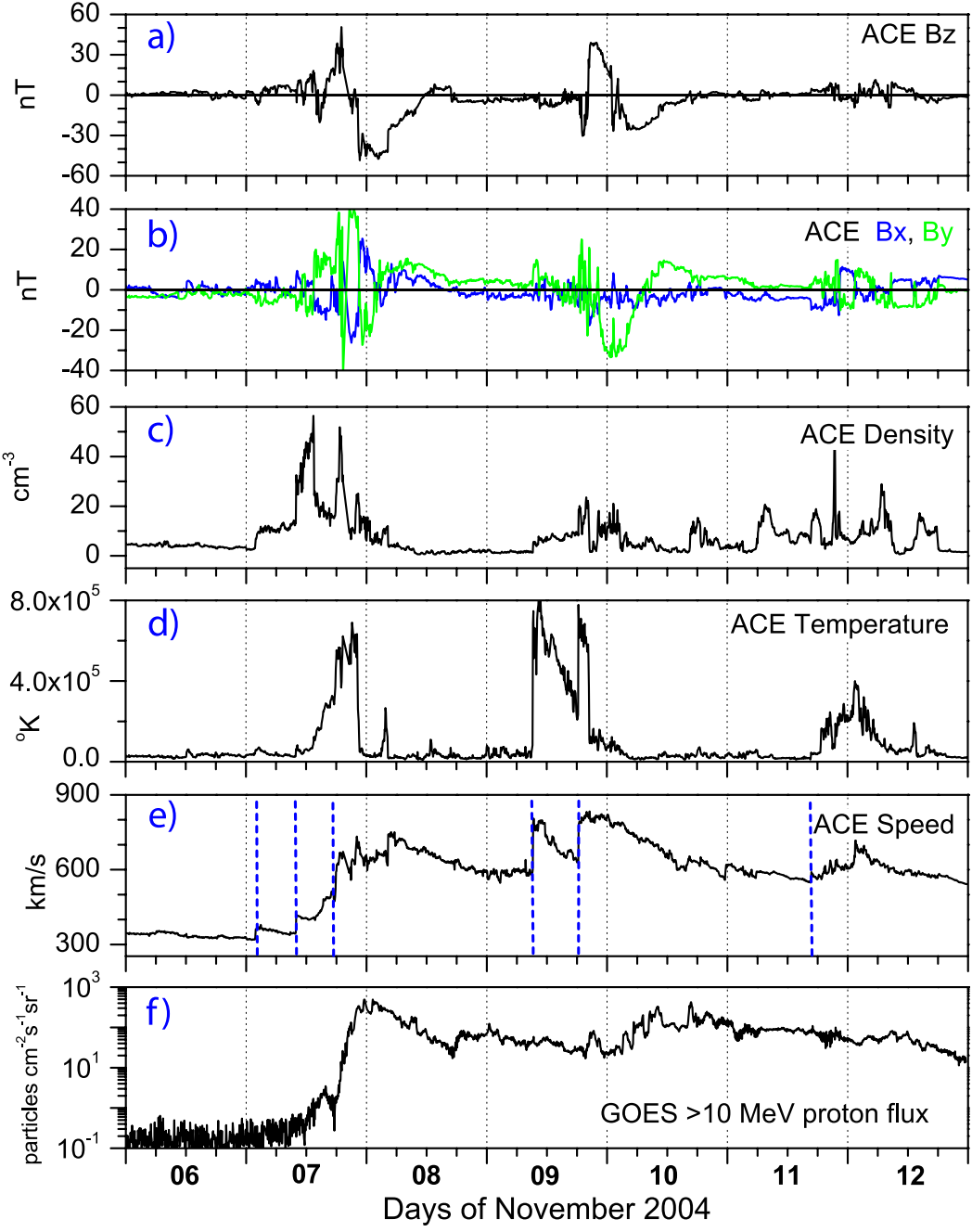

Figure 1. (a-e) ACE IMF and solar wind parameters and (f) GOES > $10 \mathrm{MeV}$ proton flux on 6-12 November.

CME (Table 1, CME 7) was probably swept up by the following fast ones and jointly resulted in the sixth shock.

[10] The CMEs most likely interacted en route from the Sun to the Earth, and the result of their interactions was seen at ACE as a compound interplanetary CME (ICME) on 7-8 November, comprising three shocks and a magnetic cloud (MC), and a second ICME on 9-10 November, comprising two shocks and an MC. Both periods contained long intervals of strong (less than $-50 \mathrm{nT}$ ) southward interplanetary magnetic field (IMF). Between these two distinct structures, the solar wind continued to have a high speed, while other solar wind parameters and the IMF became rather quiet. A third disturbed period in the interplanetary medium can also be identified on 1112 November with much smaller amplitude and no significant southward turning.

[11] The energetic ( $>10 \mathrm{MeV})$ protons started to arrive at GOES on 7 November with an abrupt increase in the daily proton flux due to the X2.0 flare (Figure 1f). The increase
10 on November was probably produced by the X2.5 flare. As several flares and CMEs were detected during a relatively short time interval, it was not always possible to discriminate between the different sources of the energetic particles. Proton fluxes stayed elevated until 17 November. The different RWCs' forecasts of the geoeffectiveness of the solar and interplanetary disturbances just described will be discussed in section 6 .

\section{Global Geomagnetic Activity}

[12] Traditionally, geomagnetic observations provide information about space weather effects on the ground. The most consistent and complete geomagnetic data are provided by INTERMAGNET observatories (http://www. intermagnet.org/index.html). The data from these observatories are used in this section to follow the development of the geomagnetic storms associated with the interplanetary sources described in section 2 . 
Table 2. Locations of the Geomagnetic Observatories

\begin{tabular}{|c|c|c|c|}
\hline Station Name & Code & Geodetic Latitude & Geodetic Longitude \\
\hline \multicolumn{4}{|c|}{ Chain 1} \\
\hline Hornsund & HRN & 77 & 15.5 \\
\hline Abisko & ABK & 68.4 & 18.8 \\
\hline Nurmijarvi & NUR & 60.5 & 24.6 \\
\hline Niemegk & NGK & 52.1 & 12.7 \\
\hline Pedeli & PED & 38.05 & 23.87 \\
\hline Hermanus & HER & -34.4 & 19.2 \\
\hline \multicolumn{4}{|c|}{ Chain 2} \\
\hline Resolute & RES & 74.7 & 265.1 \\
\hline Iqaluit & IQA & 66.7 & 291.4 \\
\hline Ottawa & OTT & 45.5 & 284.4 \\
\hline Fredericksburg & FRD & 38.8 & 282.6 \\
\hline Vassouras & VSS & -22.4 & 316.3 \\
\hline Vernadsky & AIA & -65.2 & 284.7 \\
\hline \multicolumn{4}{|c|}{ Chain 3} \\
\hline Tixie Bay & TIX & 71.6 & 129.0 \\
\hline Memambetsu & MMB & 43.9 & 144.2 \\
\hline Kakioka & KAK & 36.2 & 140.2 \\
\hline Canberra & CNB & -35.3 & 149.4 \\
\hline Macquarie Island & MCQ & -54.5 & 158.9 \\
\hline Scott Base & SBA & -77.8 & 166.8 \\
\hline
\end{tabular}

[13] Interaction of the passing ICMEs with the Earth's magnetosphere produced complex changes in the Earth's geomagnetic field. The Dst reached $-373 \mathrm{nT}$ during 7-8 November (the first ICME event) and $-289 \mathrm{nT}$ during 9-10 November (second event), and the $K p$ index was 9- for two 3-hour periods during each of the storms. Daily $A p$ indices were 140 on 8 November and 161 on 10 November. Although these indices are not exceptionally large, the morphology of the geomagnetic field variations was complicated. To describe the development of the geomagnetic situation, we use data from three approximately meridional magnetometer chains. These are as follows (see Table 2 for more detailed locations):

[14] Chain 1: Europe-Africa, Hornsund (HRN), Abisko (ABK), Nurmijarvi (NUR), Niemegk (NGK), Pendeli (PEN, non-INTERMAGNET site), and Hermanus (HER).

[15] Chain 2: America and Antarctica, Resolute (RES), Iqaluit (IQA), Ottawa (OTT), Fredericksburg (FRD), Vassuras (VSS), and Vernadski (Antarctica, AIA).

[16] Chain 3: Asia-Australia and Antarctica, Tixie Bay (TIX), Memambesu (MMB), Kakioka (KAK), Canberra (CNB), Macquarie Island (MCQ), and Scott Base (SBA).

[17] For the sake of simplicity, the first chain is assumed to be approximately at 0000 UT (within about 2 hours; for example, NUR is UT plus 2 hours), chain 2 is approximately at UT minus 5 hours, and chain 3 is at UT plus 10 hours. Figures $2 a, 2 b$, and $2 c$ show stack plots of the variations of the horizontal components (black lines) and vertical components (red lines) of the geomagnetic field during 6-12 November. The scale of the variations at the high-latitude stations (chain 1: HRN, ABK, NUR; chain 2: RES, IQA, OTT, AIA; and chain 3: TIX, MCQ, SBA) is doubled in comparison with the low-latitude stations. The numbers below the three-letter station codes in Figure 2 are corrected geomagnetic latitude calculated by using online software (http://modelweb.gsfc.nasa.gov/models/ $\mathrm{cgm} / \mathrm{cgm}$.html). Sequences of white and shaded backgrounds on the plots represent approximate local day and night, and the vertical shows 0000 UT.

[18] The development of the first cluster of geomagnetic activity proceeded approximately after 1100 UT on 7 November. At that time, the Earth's magnetosphere entered the sheath of the first ICME with its multiple fast IMF variations and at least three sudden impulses (SIs). Strong geomagnetic activity developed rapidly during the next 10-11 hours, increasing when the IMF turned southward (IMF $B_{z} \sim-20 \mathrm{nT}$ around 1400-1600 UT) and decaying when the IMF turned northward $\left(B_{z} \sim+50 \mathrm{nT}\right.$ around 1600-2230 UT) (see Figure 1a). In chain 1, located at the dayside, the auroral activity was associated with eastward ionospheric currents (ABK, Figure 2a). In chain 2, it commenced as a westward current system, changing to strong eastward current around local noon (IQA, Figure 2b). Chain 3 was at local nighttime, and westward current systems are dominant here (TIX, MCQ, Figure 2c). Rapid large variations in IMF $x$ and $y$ components with amplitudes from +40 to $-40 \mathrm{nT}$ affected the polar cap, as can be seen in rapid geomagnetic fluctuations during the local day at polar stations (RES and SBA) in both hemispheres. The southward IMF within the main part of the first magnetic cloud started to envelop the magnetosphere at about 2300 UT on 7 November, causing a rapid expansion of the auroral zone to lower latitudes and a ring current enhancement. On the dayside (chain 3, Figure 2c), the "smooth" storm main phase equatorial pattern was significantly disturbed at CNB $\left(-45.4^{\circ}\right)$ by auroral-type magnetic activity. On the evening-to-night side (chain 2), subauroral stations like OTT $\left(55.6^{\circ}\right)$ and its almost conjugate station AIA $\left(-50.4^{\circ}\right)$ have shown dominantly auroral activity, so that even the scale of the variations in Figure $2 \mathrm{~b}$ for these two stations was the same as for the regular auroral stations. On the nightside (chain 1), the westward electrojet system is centered between ABK and NUR, affecting activity at NGK $\left(48.0^{\circ}\right)$.

[19] As was mentioned in section 1, between approximately 1800 UT on 8 November and 1000 UT on 9 November, the solar wind was almost undisturbed; the existence of some interplanetary feature passing the magnetosphere can be inferred only from the high solar wind speed and slightly negative IMF $B_{z}(\sim-10 \mathrm{nT})$. At that time the activity was confined to the higher-latitude stations, indicating that the auroral zone returned to its normal position, while at low latitudes the geomagnetic field was rather quiet.

[20] The second major storm happened when the second ICME started to stream around the magnetosphere after 1900 UT on 9 November. The sharp negative excursion in IMF $B_{z}$ right after 2000 UT on this day produced a clear short duration spike in the geomagnetic field seen at 


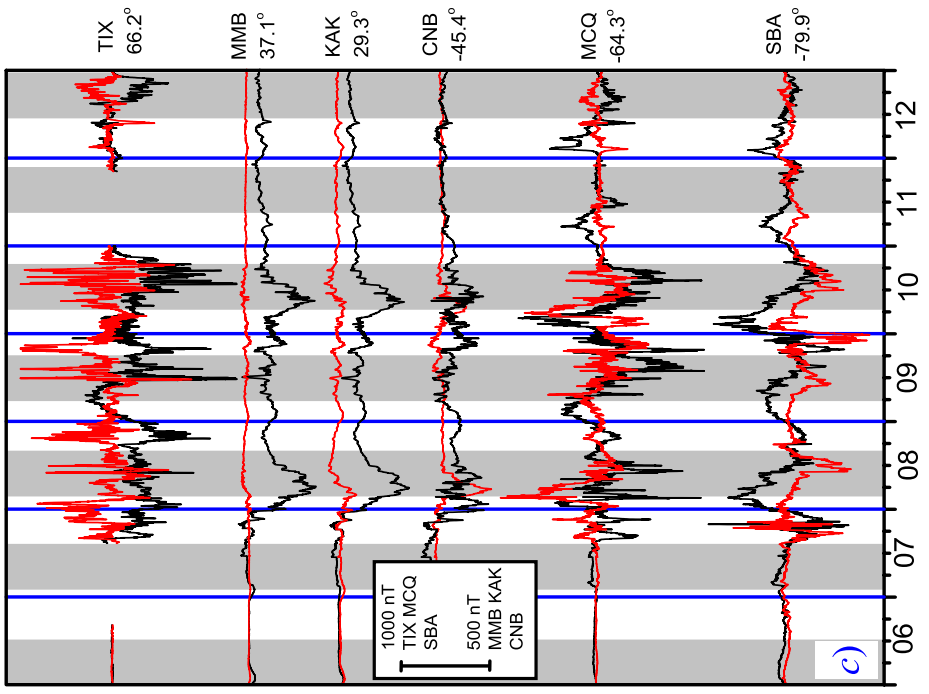

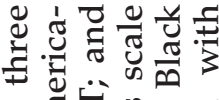

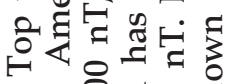
서

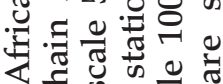

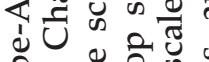

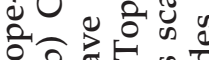

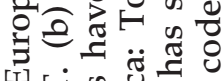

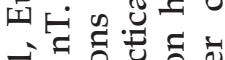

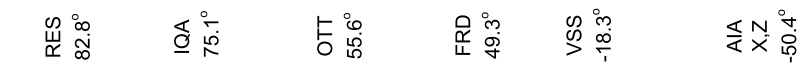
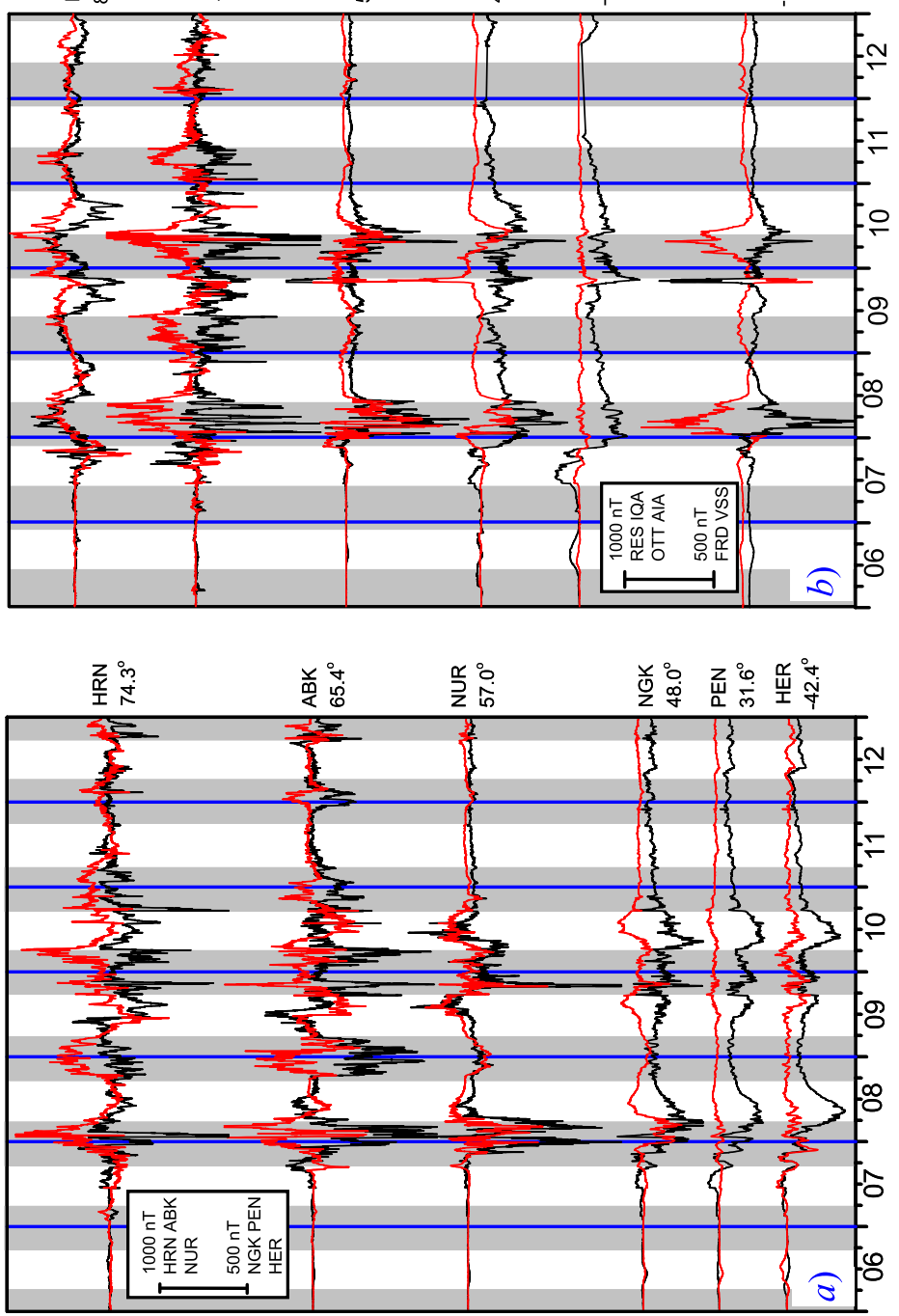

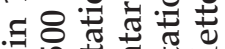

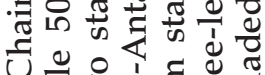

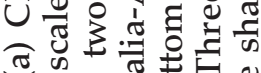
थ

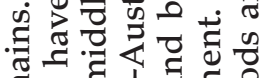

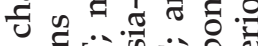

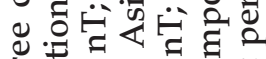
t) ఎ ช

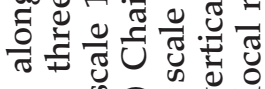

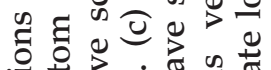

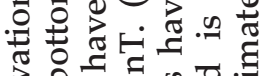

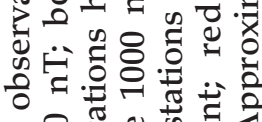
. 8 क क के

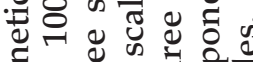

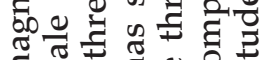

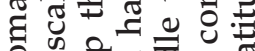

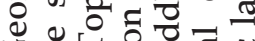

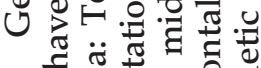
ن ข

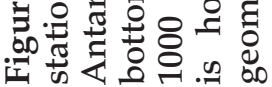



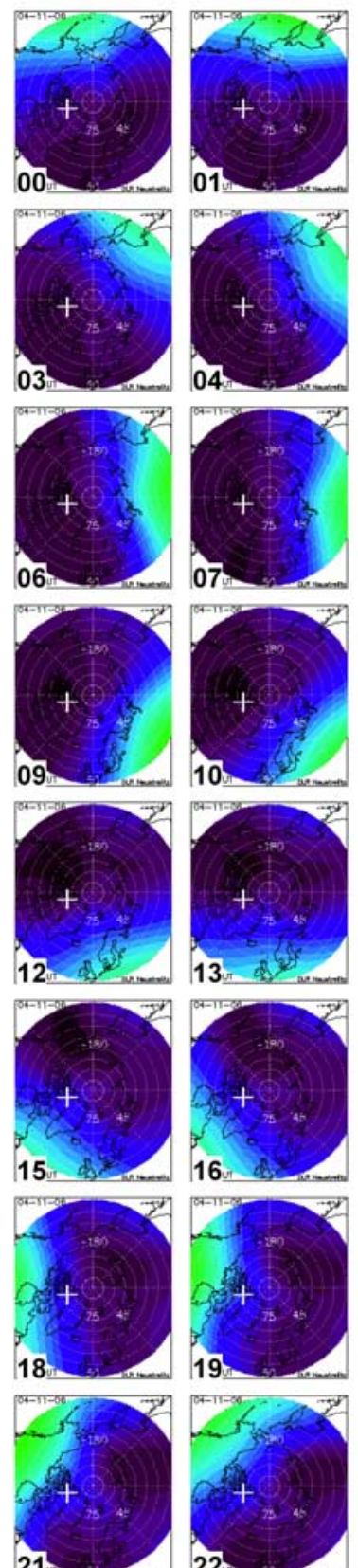

6 Nov 2004
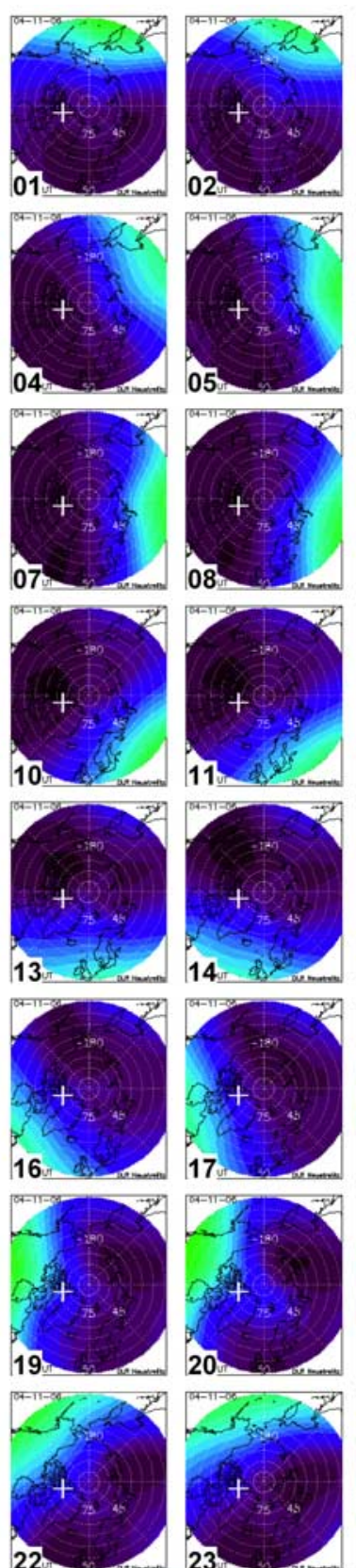

22
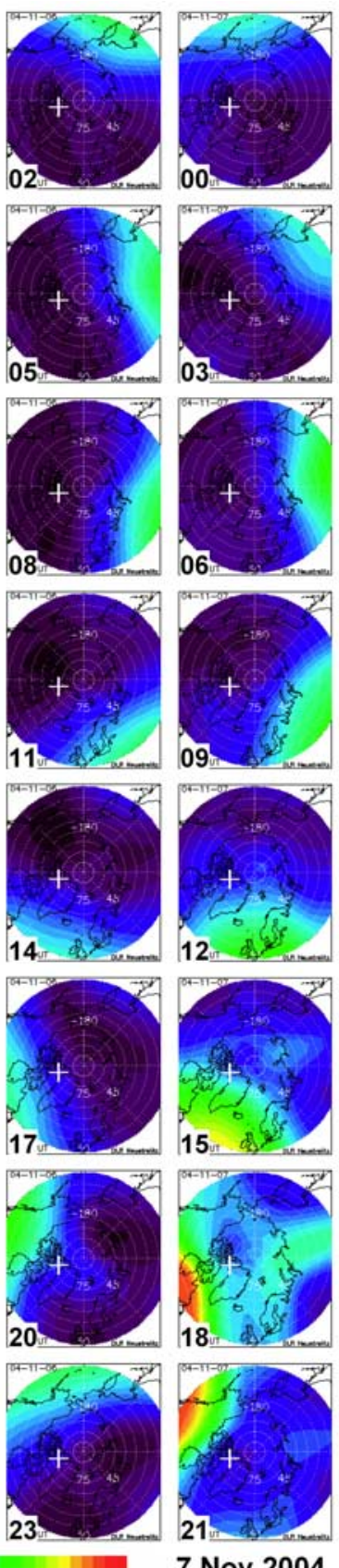
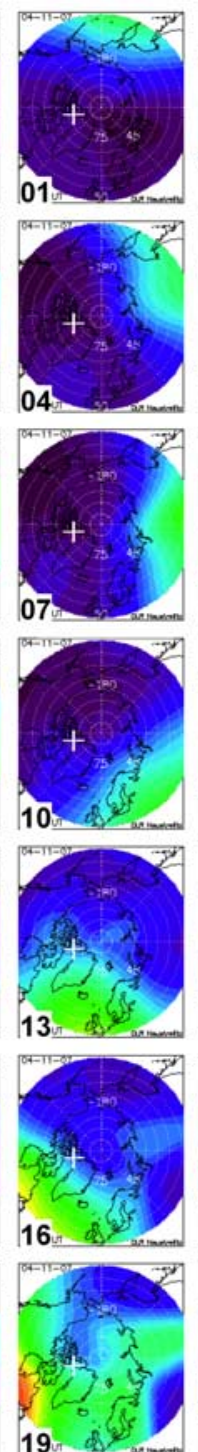

7 Nov 2004
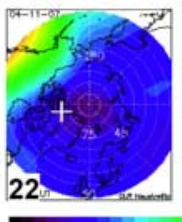
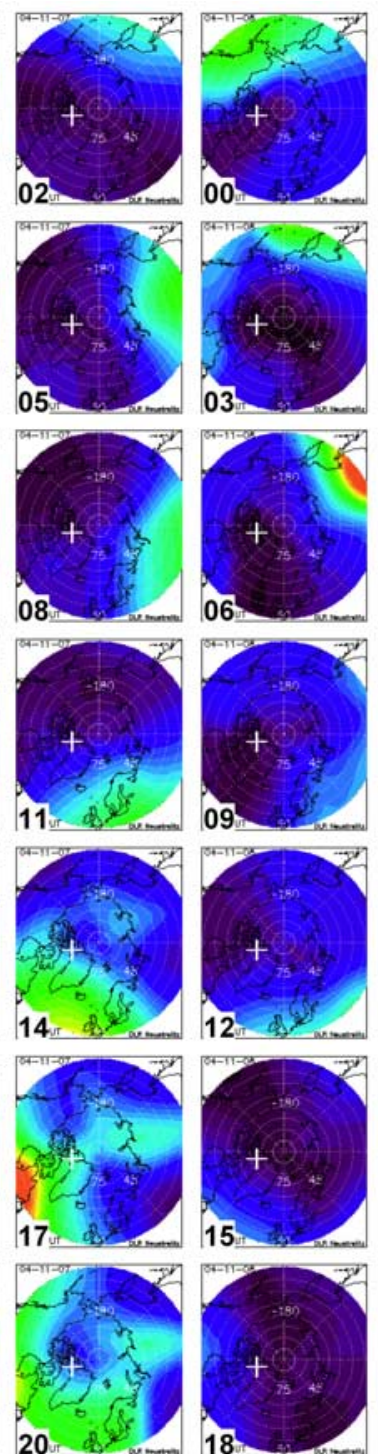
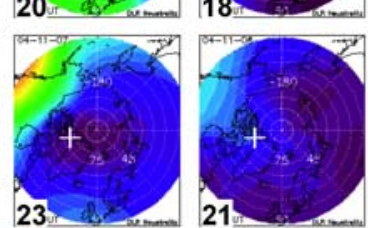

8 Nov 2004

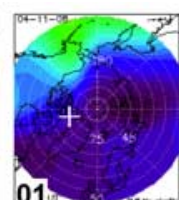

01
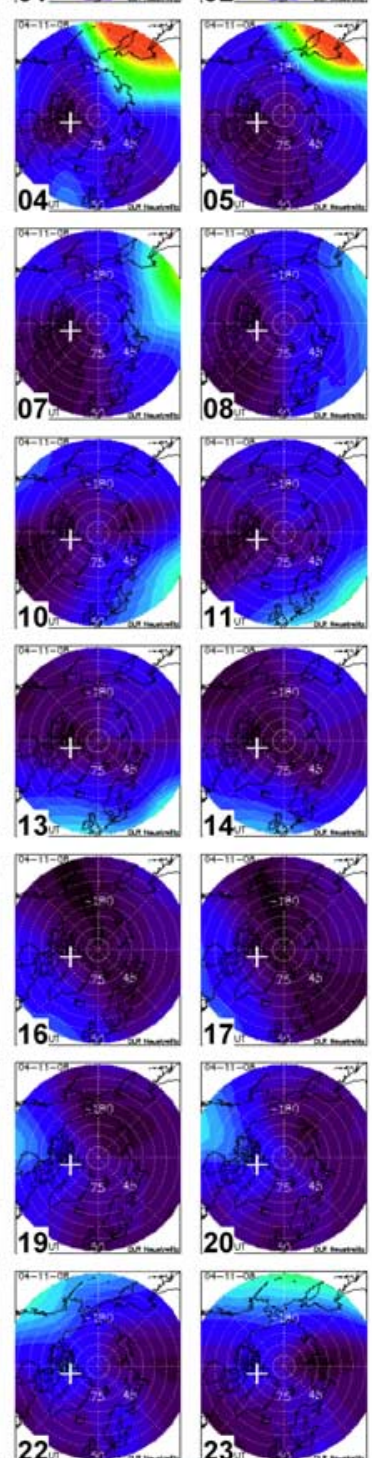

Figure 3. Hourly maps of TEC over northern polar area for 6, 7, and 8 November.

all "extended auroral" stations (with latitude $>48^{\circ}$ ). A positive turning of IMF $B_{z}$ during the time period from 2100 UT on 9 November until 0200 UT on 10 November was accompanied by a decrease in activity at "extended auroral" stations, such as OTT. After 0200 UT on 10 November, the strong negative IMF part of the second MC enveloped the magnetosphere until 1800 UT on the same day. The smooth main phase of the associated geomagnetic storm is clearly seen only below $\sim 45^{\circ}$ latitude (PEN, HER, KAK, MMB, and VSS), while other stations experienced rapid variations produced by the strong auroral current systems.

[21] For most of the magnetosphere system, the end of 10 November marked the end of significant disturbances, with the IMF returning to normal. The solar wind continued to show high speed, which might be the source of the 
remaining geomagnetic activity localized in the auroral zone.

[22] As can be seen, the geomagnetic activity was mostly defined by the parameters of the passing interplanetary disturbances and was characterized by rapid expansions of the auroral-type variations to lower latitudes. Although the Sun was active for 7 days, producing nine halo CMEs, only two geomagnetic storms occurred. Forecasting the development of the rapid and strong variations in geomagnetic activity presented a difficult task, which will be discussed further in section 6.

\section{Ionosphere}

[23] The ionospheric response is presented here using near-real-time hourly total electron content (TEC) maps (http://www.kn.nz.dlr.de/daily/tec-np/) derived from GPS data and $f_{\mathrm{o}} F_{2}$ maps (http://www.cbk.waw.pl/rwc1/ en/) based on vertical incidence sounding (ionosonde) measurements.

\subsection{Total Electron Content Maps}

[24] GNSS-based mapping of the TEC is a widely used tool for monitoring ionospheric conditions. In addition to proving useful for the evaluation of the accuracy of GPS measurements, it can also provide insight into the dynamics of ionospheric disturbances. Characteristics of the delay of the GPS radio wave propagation are obtained from the ground-receiving stations and allow the determination of slant TEC values along numerous satellitereceiver raypaths [Jakowski et al., 2002, 2005]. The calibrated "observed" slant TEC values are then mapped to the vertical by applying a mapping function, which is based on a single-layer ionosphere approximation at a height of $400 \mathrm{~km}$. Finally, the vertical TEC values are assimilated into a regional empirical model, with real values at/near the measurement points and modeled values over the areas without measurements. Such hybrid maps for 6-8 November (quiet day and the first storm) are presented in Figure 3 for the northern polar area.

[25] Indications of the storm are seen in the polar TEC maps after the second sudden impulse (SI) around 1100 UT on 7 November (Figure 3, middle), with a significant ionization region elevated above the quiet day ionization that appeared in the dayside, coinciding with strong auroral geomagnetic activity seen by magnetometers in chain 2 . On the same day, complex areas of TEC enhancements can be seen close to the pole very clearly at $1700 \mathrm{UT}$, with later (1900-2000 UT) longitudinal extension toward dusk (European) side.

[26] A period with notably complex and generally increased TEC over the entire polar region continues almost until 0700 UT on 8 November. Later, the negative part of the storm can be seen with depleted TEC values, which coincided with significant geomagnetic activity in the nightside auroral zone (Figure 2a, HRN and ABK).

[27] Although the detailed description of the TEC based on the hybrid data/model real-time maps is not complete and some of the features could be smoothed because of the modeling or averaging procedures, the complexity of the presented maps is clear evidence of a markedly disturbed ionosphere.

\section{2. $\quad F_{2}$ Region Peak Density Maps}

[28] The ionospheric parameter, $f_{\mathrm{o}} F_{2}$ (ordinary ray critical frequency of $F_{2}$ layer), is frequently used for providing communications support. The complexity of the ionospheric disturbances during the period of study is supported further by $f_{\mathrm{o}} F_{2}$ maps in the European, Japanese, and Australian regions. They have been created in near real time by RWC Poland, using the instantaneous mapping model [Stanisławska et al., 2001; Stanisławska and Zbyszyński, 2001], on the basis of "URSIgram" coded data exchange of ionosonde data (http://www.ises-spaceweather.org). Ionospheric $f_{\mathrm{o}} F_{2}$ maps (Figure 4) show the spatial snapshot at 1200 UT for each day during 6-10 November. European maps cover $90^{\circ}$ in latitude by $100^{\circ}$ in longitude, Australian maps are $70^{\circ} \times 80^{\circ}$, and Japanese maps are $24^{\circ} \times 24^{\circ}$.

[29] With the first ICME enveloping the magnetosphere, the ionospheric effects are seen as a general increase of the ionization. On 7 November at $1200 \mathrm{UT}, f_{\mathrm{o}} F_{2}$ values are significantly higher than normal (for example on 6 November), particularly in the local evening (over the northeast part of Europe). This is associated with the second SI on 7 November (Figure 1). In the evening-midnight sector (Australian and Japanese maps), the ionosphere shows reduced $f_{\mathrm{o}} F_{2}$ values and larger cross-equatorial latitudinal gradients. On 8 November, the negative phase of the first storm is seen during daytime conditions in Europe, which lead to a decrease in $f_{\mathrm{o}} F_{2}$. At the same time, the evening-midnight side of the near-equatorial ionosphere shows complex patterns in the $f_{\mathrm{o}} F_{2}$ contours over Australia with gradients in the east-west direction (near latitude $10^{\circ}$ ) and overall increase in $f_{\mathrm{o}} F_{2}$, which is also clearly seen over the Japanese archipelago.

[30] Around 1200 UT on 9 November, the sheath of the second ICME with its high-temperature high-speed solar wind but relatively small IMF $B_{z}(\sim-10 \mathrm{nT})$ interacted with the magnetosphere. Maps of 9 November in Figure 4 show a second, less pronounced, and more uniform overall increase in $f_{\mathrm{o}} F_{2}$ over Europe (dayside). The enhancement in near-equatorial evening hours can be seen in the Australian map with a trough region between latitudes $-20^{\circ}$ and $-50^{\circ}$ and auroral enhancement around $-65^{\circ}$ latitude, which coincided with the extension of the auroral oval seen in geomagnetic data (Figure 2c). Maps for Japan (approximately 1900-2200 local time, 9 November) show an overall nighttime decreased $f_{\mathrm{o}} F_{2}$. During the next day, steep gradients appear because of the very unstable stormy situation apparent in all maps.

[31] The mapping procedure requires significant interpolation and cannot be precise in the case of the sparse data points during high temporal variability. Unfortunately, this is the situation during the storm periods. For instance, 

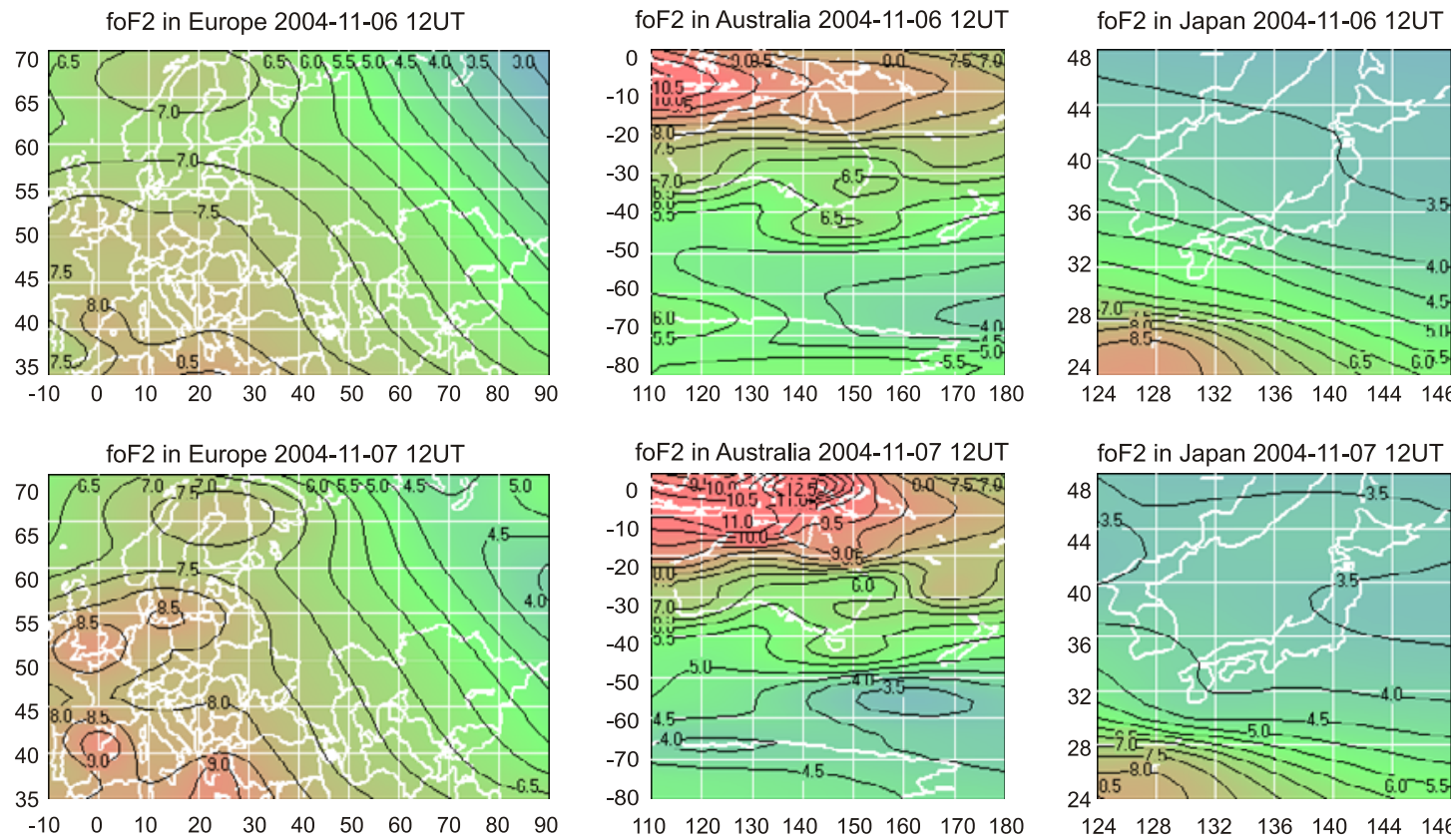

foF2 in Australia 2004-11-07 12UT
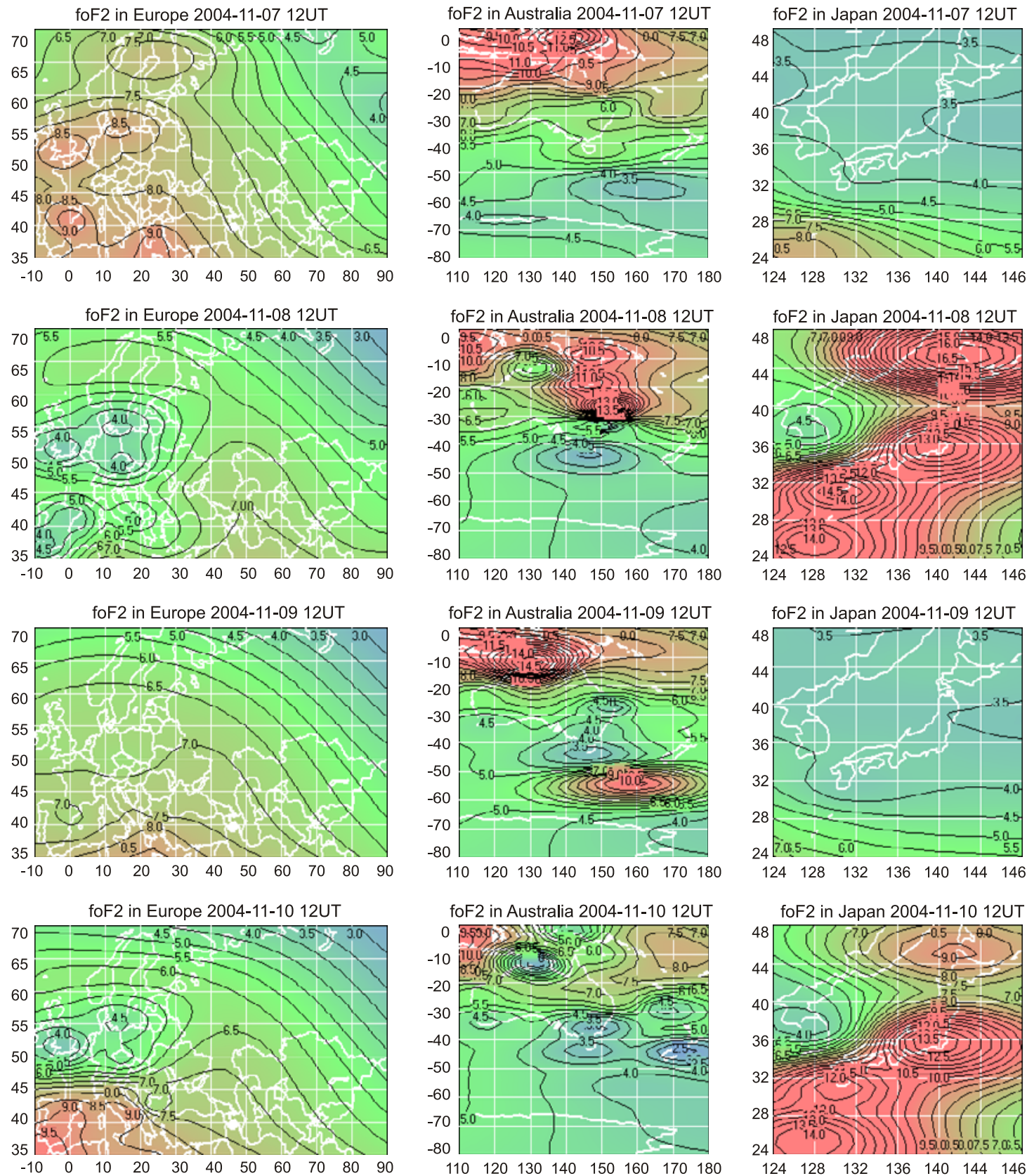

Figure 4. Maps of $f_{\mathrm{o}} F_{2}$ at $1200 \mathrm{UT}$, created for the sequence of 5 days, 6-10 November, for the European, east Asian, and Australian regions. 


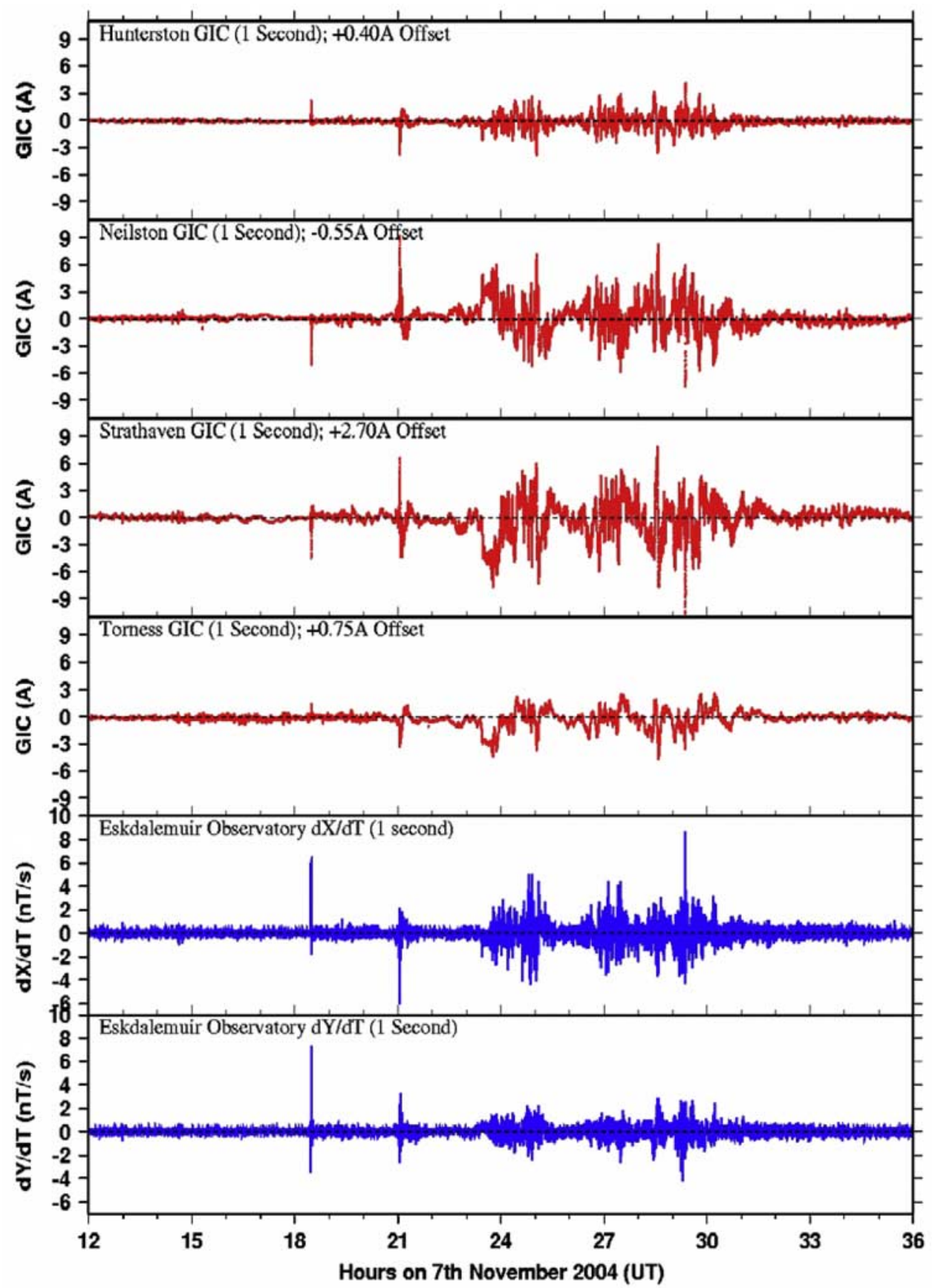

Figure 5. Measured GIC levels in the Scottish Power network and Eskdalemuir Observatory data from noon 7 November until noon 8 November.

high-energy proton fluxes associated with the X2.0 flare on 7 November, 1606 UT (Table 1) produced a polar cap absorption (PCA) event that continued until 13 November. During these days, no $f_{\mathrm{o}} F_{2}$ observations could be made at locations within and near the auroral oval, which led to no data from Scandinavian ionosonde stations. Furthermore, on 8 November a severe particle absorption event produced a 6-hour data gap for Hobart that, with the absence of data for 10 November from Macquarie Island, significantly reduced the reliability of the maps for the Australian area on these days.

\section{Effects on Power Systems and Pipelines}

[32] One of the important effects of geomagnetic storms is their impact on ground-based technological systems. In brief, variations of the geomagnetic field induce electric 

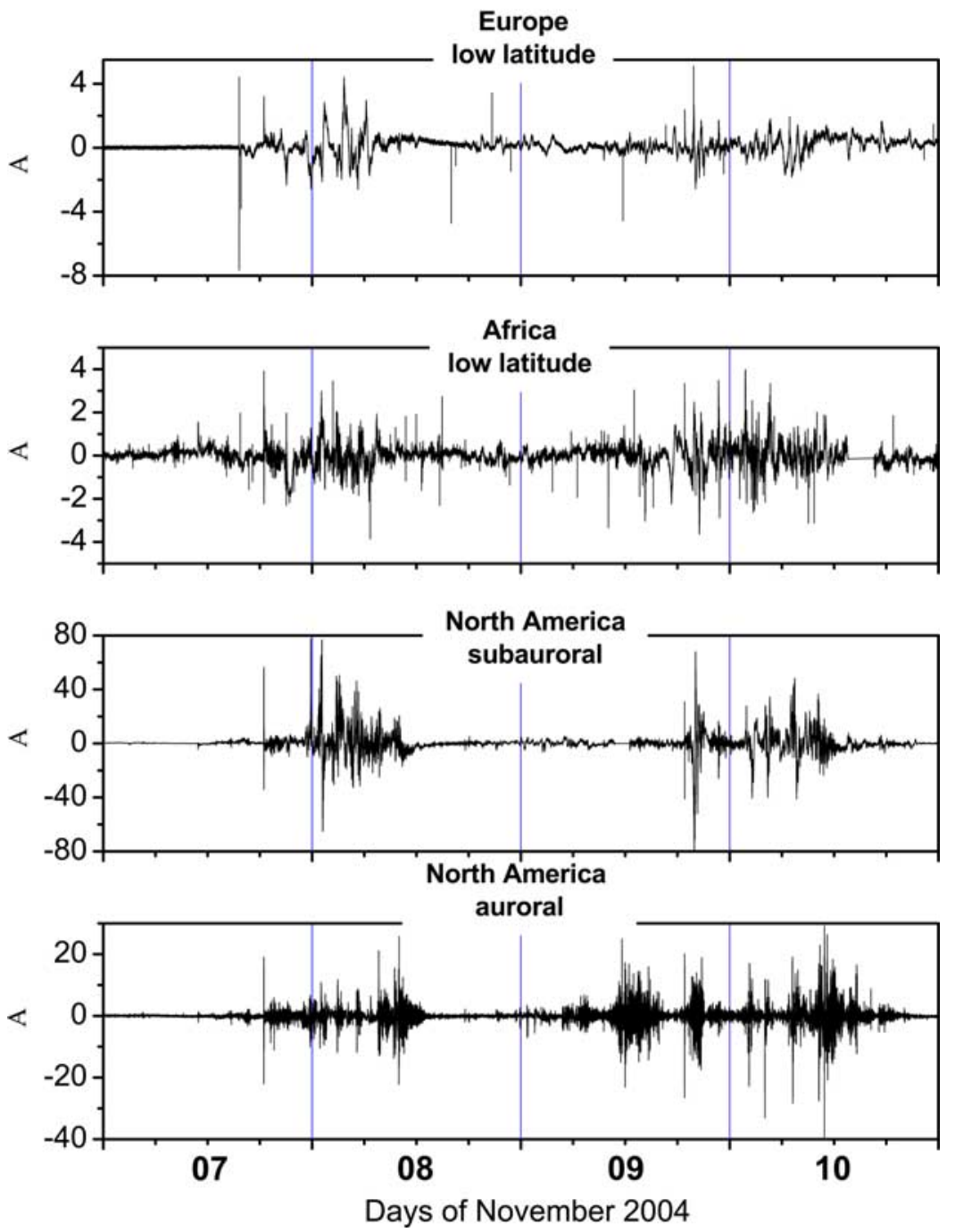

Figure 6. GIC measured at specific sites of four power systems, located in different regions for four disturbed days in November 2004.

currents in the ground and any grounded or closed-loop conductive structures, such as power grids or pipeline networks. During geomagnetic storms, geomagnetically induced currents (GIC) can be large enough to disturb the regular operations of these systems. After the famous blackout of the Hydro Quebec power system in 1989, GIC monitors were installed at many locations in different power systems around the world. As well, power companies use global $(K p)$ and local geomagnetic indices (hourly ranges, hourly standard deviations, or rate of change) to be informed about current geomagnetic activity.

[33] As an example, the GIC monitoring data from four sites on the Scottish Power grid and local geomagnetic derivatives from the closest geomagnetic observatory in Eskdalemuir are presented in Figure 5. All GIC data show a clear relationship to the local geomagnetic field rate of change (north component). In general, the GIC levels during the first geomagnetic storm (7-8 November) were not particularly large, with a maximum of $6 \mathrm{~A}$, compared with 42 A during the "Halloween" storm of October 2003 [Thomson et al., 2005]. At the same time, a significant level of GIC up to $109 \mathrm{~A}$ in Sweden has been reported during the 9-10 November 2004 storm [Lundstedt, 2005], although this was most likely caused by the specific type of power grid components rather than the severity of the local geomagnetic conditions.

[34] Regular GIC recordings at the sites belonging to the different power grids around the world are continuously provided by the Sunburst Program of the Electric Power Research Institute. Examples from different power grids in Europe, Africa, and North America (one recording site from each power grid) are presented in Figure 6 for $7-10$ November 2004. It can be seen that the largest levels (up to $80 \mathrm{~A}$ ) were observed at the site located in the subauroral 


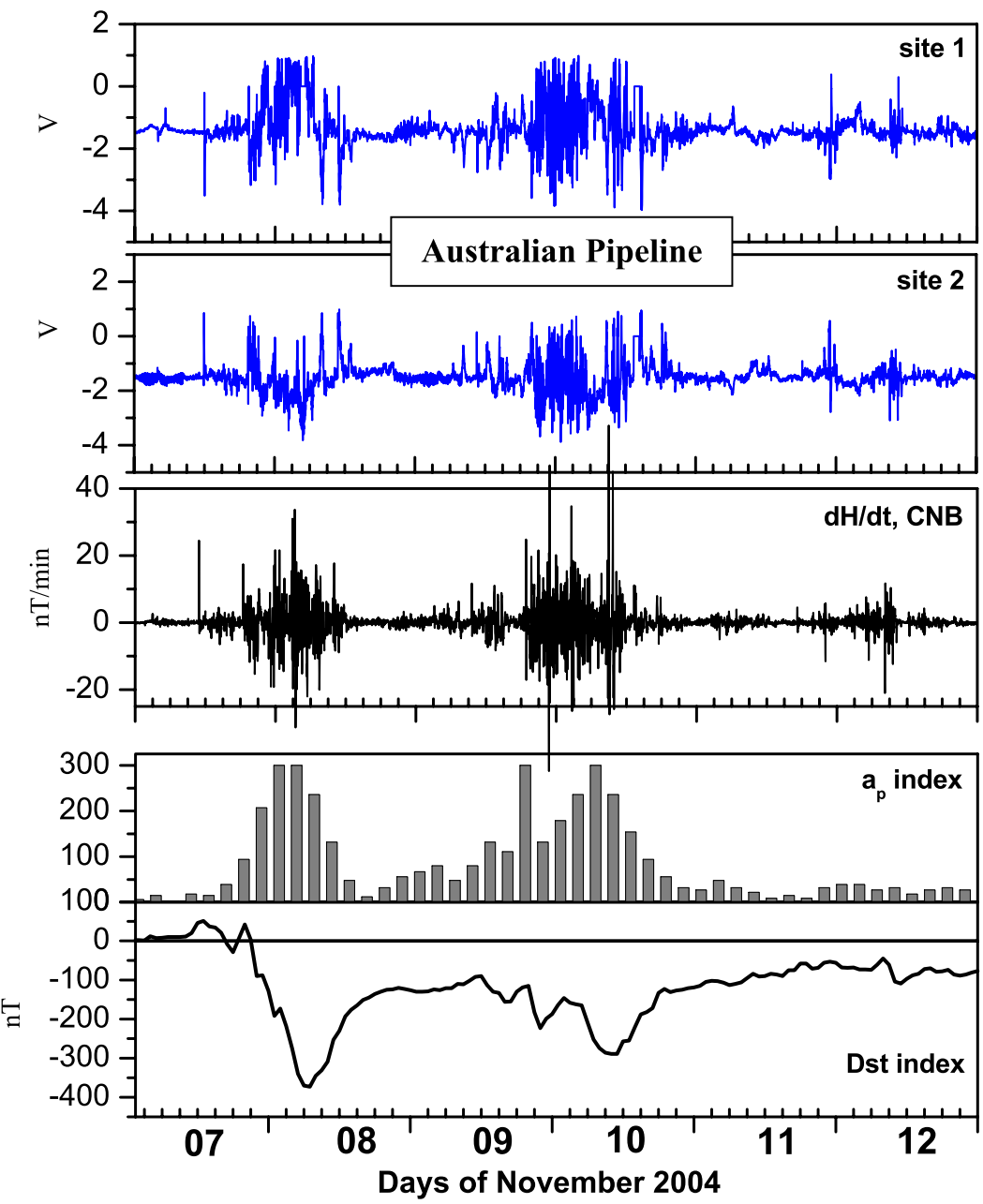

Figure 7. Pipeline voltage at two sites, time derivative of the horizontal component of geomagnetic field at $\mathrm{CNB}$, and ap and Dst indices for 7-12 November.

zone of North America, due to the specific details of the power network where the recordings were made. For proprietary reasons, the exact locations of these sites are unavailable.

[35] Although GIC in the power grids were not large enough to cause problems, exceptionally large voltages were observed in a gas pipeline in eastern Australia (Figure 7) during the whole period of 7-12 November. In quiet conditions, variations of pipeline voltage stay between $-1 \mathrm{~V}$ and $-2 \mathrm{~V}$, which is the operating range for corrosion protection. When the pipeline voltage is more positive, corrosion rates are larger, which leads to reduction of the pipeline lifetime and increased risk of unexpected leakage. As can be seen in Figure 7, during the storms of 7-8 November and especially 9-10 November, the amplitude of the voltage fluctuations at site 1 of this pipeline was up to three times larger, producing long periods of time with voltage out of the prescribed protection range.
[36] Another negative aspect is that the voltage readings during geomagnetic storms can be misinterpreted as produced by corrosion pitting in the pipeline steel. This can make it necessary to perform the costly procedure of a detailed pipeline survey. Thus the monitoring and forecast of the geomagnetic storms are an important part of mitigation of the GIC effects on pipelines.

[37] Comparisons of pipeline voltage fluctuations with rate of change of the local geomagnetic filed and with the ap and Dst index (Figure 7) show that the voltage variations more closely follow the variations of the local geomagnetic field. Therefore indices based on local geomagnetic field variations are more reliable than global indices for interpretation and forecasts of the GIC [Trichtchenko and Boteler, 2004].

\section{Forecasts}

[38] In the previous sections, we presented the solar, interplanetary, and geomagnetic conditions during the 


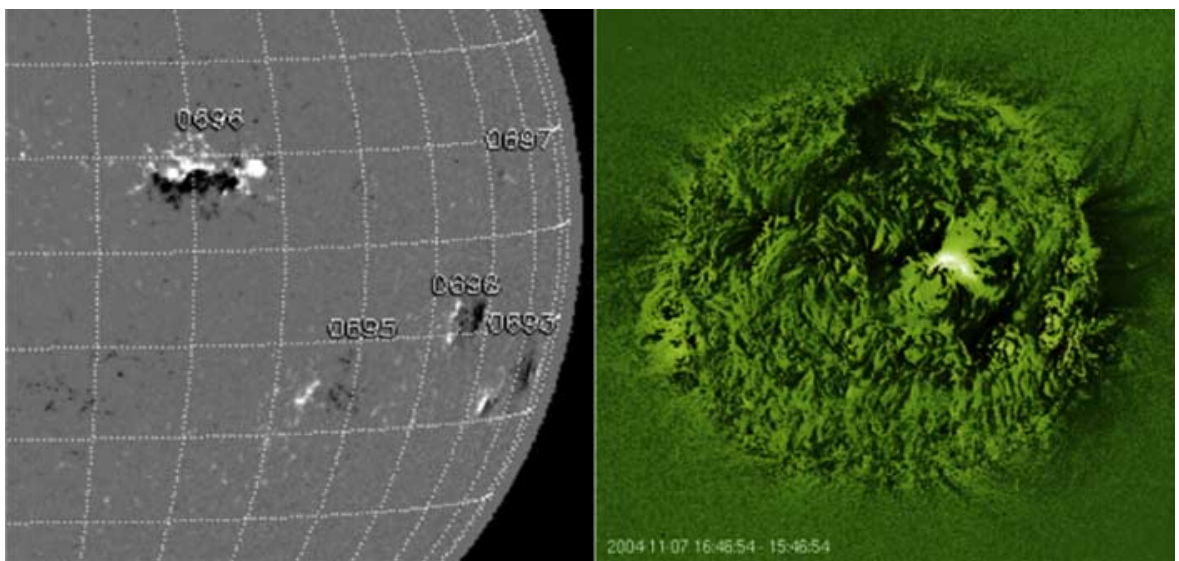

Figure 8. (left) Part of a SOHO/MDI magnetogram obtained at 2228 UT on 7 November (from http://www.solarmonitor.org). (right) SOHO/EIT difference image showing the coronal dimming areas after the eruption on 7 November. The last preeruption image taken at 1546 UT was subtracted from the image taken at 1646 UT.

progression of the space weather events of $7-12$ November 2004 and their effects on ionospheric parameters important for the users of communication and navigation systems and on geomagnetically induced currents in power systems and pipelines. This section describes the forecasting experience of four different RWCs during the time of the November 2004 events. Each of the RWCs delivers different types of space weather forecasts defined by the
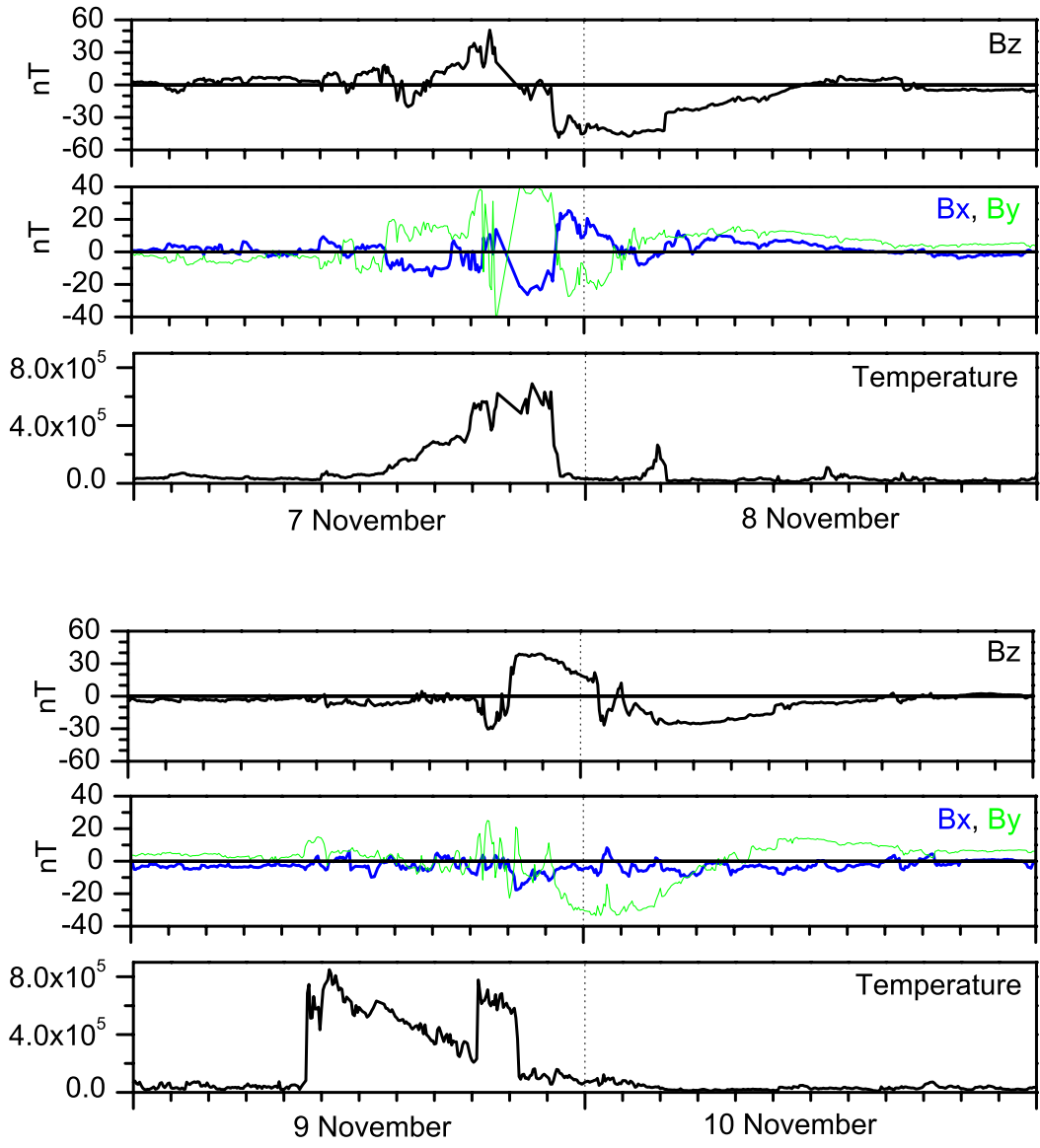

Figure 9. (top) ICME characteristics during 7-8 November as seen in ACE solar wind data. (bottom) Same as the top plots but for 9-10 November. 
requirements of their local users and therefore demonstrates different emphasis in their analysis.

\subsection{Forecast of the ICME Magnetic Field Orientation}

[39] The most important part of any forecast is an evaluation of the geoeffectiveness of a CME and its time of arrival at the Earth. The geoeffectiveness is directly dependent on the orientation of the magnetic field of the ICME, and here we present the forecast of the IMF orientation during the November events made by RWC Belgium.

[40] The photospheric magnetic field of the CME source region often has a bipolar configuration. According to the so-called "standard model" of solar eruptions (see discussion by Hudson and Cliver [2001]), the axis of the ejected flux rope is aligned with the photospheric neutral line. Assuming that this orientation does not change during the eruption, one can determine the inclination of the axis of the interplanetary flux rope [Marubashi, 1986; Yurchyshyn et al., 2001; McAllister et al., 2001]. If the shear of the magnetic field can be determined (for example by looking at the posteruption arcade in the SOHO EIT data), the direction of the magnetic field in the flux rope can be estimated.

[41] The main neutral line in AR 10696 was stretched in the east-west direction during its whole passage across the solar disc (see photospheric magnetogram in Figure 8, left), with the positive polarity (shown in white) to its northern side. This signifies that the leading edge of the large-scale magnetic field of the erupting flux rope is expected to be southward. As was reported in the real-time alert message issued by RWC Belgium on 5 November, 0906 UT, "... The arrival of an interplanetary disturbance corresponding to these CMEs is expected on November 7-8. The occurrence of a strong geomagnetic storm is possible, as the ejected interplanetary flux rope will probably have the leading southward magnetic field." As can be seen from ACE data (Figure 9), the magnetic cloud that begins to be observed around 2200 UT on 7 November and ends around 1700 UT on 8 November was of the south-east-north type; that is, its magnetic field was rotating from south to north with an axial field directed to the east [Bothmer and Shwenn, 1998], in agreement with the prediction.

[42] The situation with the second magnetic cloud was different. The orientation of the neutral line of AR 10696 had not changed; therefore a magnetic field with south to north field rotation was expected. However, the magnetic cloud detected by ACE on 9-10 November (see Figure 9) had an orientation between NWS and WSE types. A more detailed inspection of EIT dimmings after the CME of 7 November (Figure 8, right) provides a possible reason for this. Except for the dimmings in AR10696 and around it, another large dimming is present, to the southwest of the active region. It is associated with the sheared transequatorial loops connecting AR 10696 and AR 10695. The chromospheric observations in $\mathrm{H} \alpha$ show that a filament was situated under these loops, and it disappeared on 7 November. The orientation of the photospheric magnetic field around this filament shows that the filament flux rope was of the NWS type with the inclination of $45^{\circ}$ to the east-west direction and was too complicated to be represented by a simple cylindrical flux rope. This is a possible reason why the forecast for the orientation of the second magnetic cloud (based on a simple model) was not correct.

\subsection{Forecasts of the Geomagnetic Field}

[43] Even if the orientation of the interplanetary magnetic field in an ICME is known, the forecast of the arrival time of the part with a southward directed magnetic field and its amplitude needs to be significantly improved. As has been shown in the forecasts quoted in section 6.1, the start time and the amplitude of the geoimpact of the complex disturbance have mostly been forecast qualitatively. Two other RWCs represented by the authors of this paper (Australian and Canadian) provided more quantitative forecasts of the geomagnetic activity and hence estimates of the time and amplitude of the geoimpact produced by the ICME.

[44] Global geomagnetic activity forecasts (daily $A p$ index) were produced by RWC Australia. For the time period investigated, these were based first on the flaring activity of AR 10696 on 3-4 November (Table 1), which was judged likely to increase magnetic activity at the Earth, and alerts were issued. The full-halo CME and associated flares on 6 November triggered a forecast of $A p=60$ for 8 November that was accompanied by geophysical and ionospheric storm warnings for the whole period of 9-11 November. In reality, the anticipated geomagnetic storm started 12 hours earlier than expected (late on 7 November, as was shown in section 3 ) and its intensity ( $\max A p=140$ ) was significantly underestimated. Subsequent magnetic activity was initially expected to fall back to nonstorm levels by 11 November, but the alert level was upgraded after the $\mathrm{X} 2$ flare and full halo CME on 7 November was reported, which may have been the source of renewed severe activity of 9-10 November (see section 3 for details).

[45] Severe storm levels were forecast for 11 November, because a number of statistically significant storm indicators, as was shown in section 2, suggested a potential risk: full halo CME on 9 November (Table 1, CME 8) and the $\mathrm{X} 2 / 3 \mathrm{~B}$ flare on 10 November accompanied by a very fast halo CME (Table 1, CME 9), although possibly directed off center with respect to the Earth; there was a strong type II/ IV radio emission; a parallel-ribbon flare, and a Castelli $\mathrm{U}$ burst were reported. However, the expected high levels of geomagnetic activity did not occur. Since AR 10696 was westward of the statistically optimum region on the Sun for storm potential, possibly this forecast could have been downgraded. Given the indicators above, it could be argued that the forecaster was placed in a difficult position. At issue here are whether it would have been reasonable to expect the forecaster to make a different 


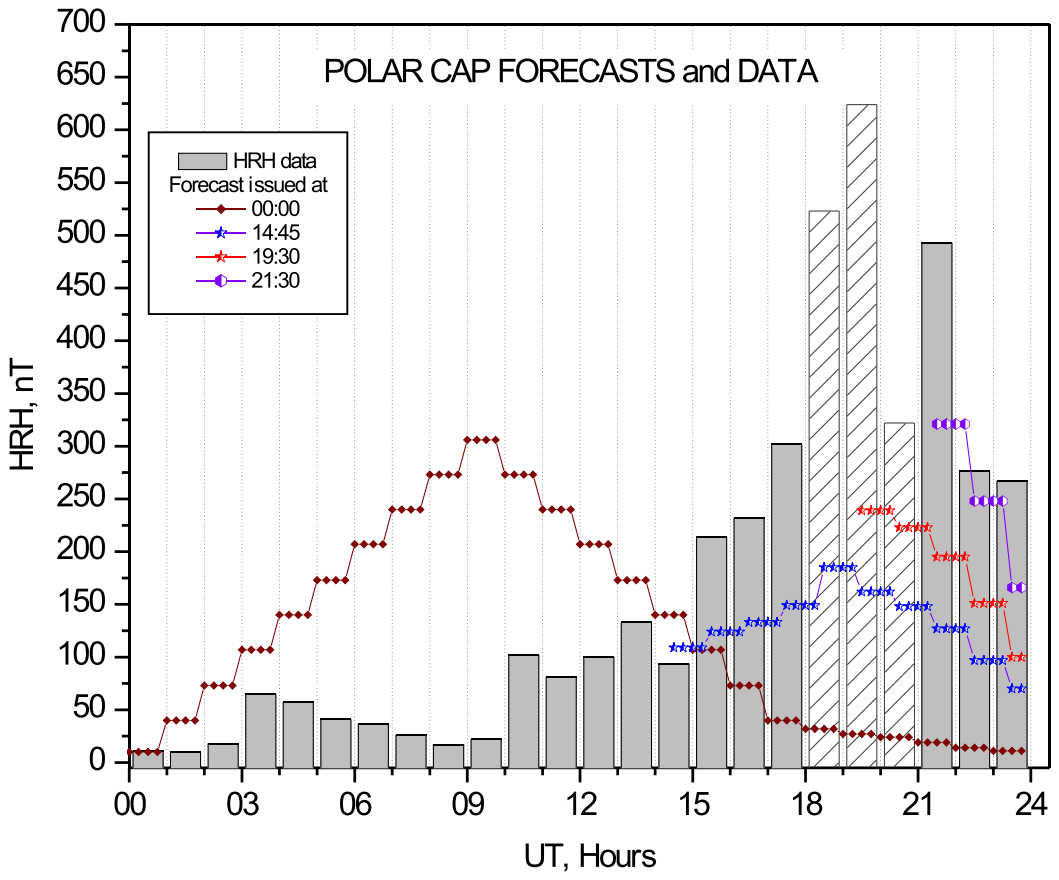

Figure 10. Hourly range indices for RES observatory on 7 November. Gray bars represent data, hatched bars indicate data gaps, and colored lines show forecasts.

forecast given the information available at the time and what extra information would be helpful in the future.

[46] Geomagnetic forecasts, produced by RWC Canada, are for the local geomagnetic activity, in terms of the hourly range index (hourly range of the horizontal component, HRH). Current and expected geomagnetic activity forecasts are five semiquantitative levels (quiet, unsettled, active, stormy, and major storm) defined on the basis of the past, current, and expected HRH. They are issued automatically every $15 \mathrm{~min}$ for each of the 13 observatories across Canada and also for spatially averaged "zonal" geomagnetic activity: the polar, auroral, and subauroral zones.

[47] These forecasts are based on the information on solar and solar wind conditions (discussed in section 2) and the average statistical pattern of the local geomagnetic activity. They also incorporate real-time geomagnetic data supplied by each Canadian observatory. The examples of the geomagnetic activity during the November 2004 event were discussed in section 3 and are shown for three Canadian observatories in Figure 2b (RES, IQA, and OTT). Here we discuss in detail the forecasts for the polar cap area, represented by the RES observatory. The five levels of polar cap geomagnetic activity are defined as "quiet" when HRH at RES are below $50 \mathrm{nT}$, "unsettled" for ranges of 50-100 nT, "active" for 100-175 nT, "stormy" for 175-450 nT and "major storm" for HRH > $450 \mathrm{nT}$.

[48] The first storm on 7 November was expected for all three zones in Canada, with times of maximum activity initially predicted to be a half day earlier, and was adjusted later, during the event passage, by incorporation of realtime geomagnetic data. Examples of the polar zone forecasts issued at the different times of the day (colored lines) and the real hourly indices, calculated later (gray bars) are shown in Figure 10. An increase in the geomagnetic activity was forecast at 0000 UT (brown line), with a maximum at 0900 UT (10.5 hours earlier than observed) based on the expected time of arrival of the CMEs of 4 November estimated from field-of-view (FOV) speed (Table 1, CME 4). The maximum activity was overestimated by three levels, i.e., "stormy" instead of "quiet." Later in the day, because the expected CME did not arrive, the forecast issued at 1445 (blue line) follows the statistical pattern of the daily activity for this station (nighttime increase in activity). For that period of time, the geomagnetic activity forecast was "active," while in reality it ranged from "stormy" to "major storm." From 1800 to 2100 UT, there was a gap in the real-time data supplied by the only observatory in the polar cap (RES). As seen from Figure 10, forecasts issued at 1930 incorporated the statistical pattern and data received before 1800 and could have followed the real activity better if the data were not missing. As soon as the geomagnetic data stream was restored, a forecast was issued at $2130 \mathrm{UT}$ and shows very good agreement with the data. At that time, the information about the halo CME of 6 November had not been incorporated in the forecast (the input coded UCMEO message came only on 8 November). Also, because of the large FOV speed of the full-halo CME of 7 November, 

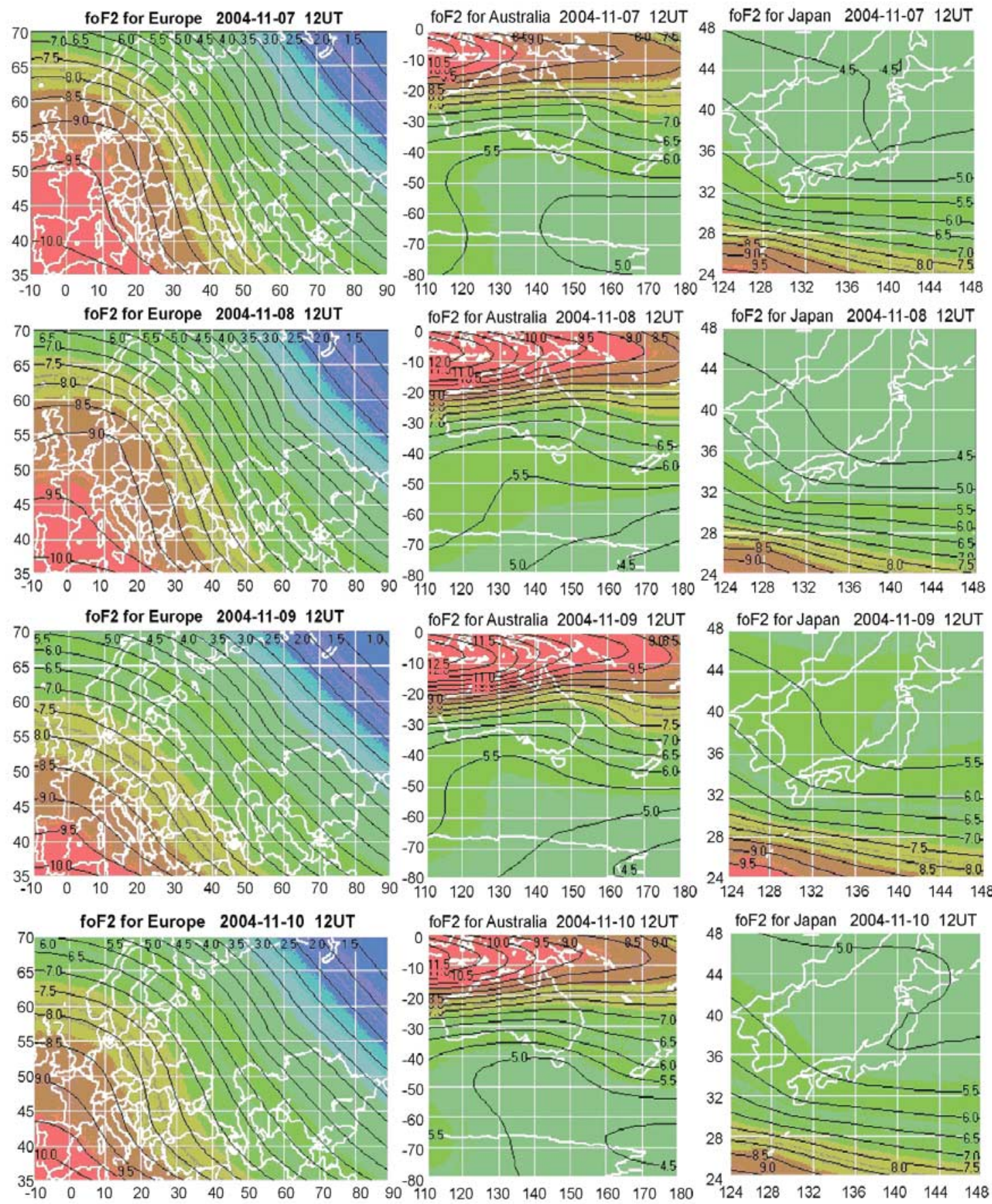

Figure 11. Maps of predicted $f_{\mathrm{o}} F_{2}$ over (left) Europe, (middle) Australia, and (right) Japan at 1200 UT for 7-10 November.

the second increase in geomagnetic activity was forecast for the second half of 8 November, while in reality it happened 1 day later.

[49] In general, for the period of the first geomagnetic storm, 7-8 November 2004, the daily forecast patterns of hourly range indices for all three zones differed significantly from their observed values. The forecasts were adjusted automatically as real-time data became available, making forecasts of 1-3 hours ahead in time more reasonable. The most extreme "storm watch" conditions issued by RWC can only be triggered by real-time geomagnetic data, which helps its reliability. The "storm watch" conditions have been issued properly for the duration of both storms. Later, in forecasts for 10-11 November, 
the geomagnetic activity in all zones and observatories was slightly overestimated because of the expected effects of the solar activity on 9 and 10 November as has been described for the Australian RWC forecasts.

[50] Certain improvements in the forecasts of the local geomagnetic activity in the polar zone could have been done if at that time the real-time data from more than one observatory would be supplied, as it is now. However, this will only help in the forecasts of up to 6 hours ahead; in order to get more reliable forecasts further into the future, reliable models of the CME interactions and propagation would be the most helpful.

\subsection{Ionospheric Predictions}

[51] Ionospheric conditions and global ionospheric forecasts were provided by two RWCs. A set of global ionospheric forecasts and warnings was issued by RWC Australia. Because of the flare potential of AR 10696, high-frequency (HF) fadeout warnings were issued for the period 4-13 November. On 5 November, an ionospheric storm was predicted for 8 November, and on 6 November a significant storm was being forecast for 9 November. The storm period was extended when the SI on 7 November occurred, and subsequently, depressed ionospheric conditions were forecast out to 14 November. These forecasts were reasonable but did not anticipate the severity of the storm adequately. Height rises in the ionosphere late on 7 November and flowing into 8 November resulted in sharp decreases in HF communication capability throughout the Australian region, especially at high latitudes. This was followed by severe depressions, up to $50 \%$, that continued until 13 November. During this period, ionospheric variability was greater than usually observed, with larger than usual ionization gradients across Australia. These effects led to major problems for all HF users.

[52] Detailed operational ionospheric maps were forecast 24 hours ahead by RWC Poland. These maps of the $f_{\mathrm{o}} F_{2}$ forecasts for three regions (Figure 11) can be compared with post factum maps presented in Figure 4 and described in section 4. Comparison of $f_{\mathrm{o}} F_{2}$ in the European area shows that the minimum value forecast was $1.0 \mathrm{MHz}$ (for 8 November), while the minimum mapped in Figure 4 was $3.0 \mathrm{MHz}$; the maximum value forecast for the same day was $10 \mathrm{MHz}$, while the mapped value in Figure 4 was only $4 \mathrm{MHz}$ (depletion). The forecast would not be expected to reproduce all the complex detail that characterizes the disturbed ionosphere. The large $f_{\mathrm{o}} F_{2}$ patterns show better coincidence between the forecast and post factum mapped values over Australia than over Europe. For the Japanese area, the largest deviations in forecasts are during 8 and 10 November.

[53] Although the $f_{\mathrm{o}} F_{2}$ forecast and map generation methods proved their high quality in several cases and in statistical studies [Stanisławska et al., 2001; Stanisławska and Zbyszyński, 2001], there are difficulties in specific operational applications such as during the event described, with the extremely high intensity and rapidness of the disturbances. The lack of data from some stations, mentioned in section 4, also decreased the accuracy of both the predictions and post factum maps.

\section{Conclusions}

[54] Analysis of the space weather conditions for the November 2004 event, based on real-time data, shows the initial complexity of the solar sources and continuous interactions of the multiple (at least nine) CMEs during their propagation through the solar wind. These interactions led to the creation of only two complex geoeffective ICME structures that reached the Earth on 7-8 and 910 November, while the later, faster CMEs appeared to be nongeoeffective. Ground observations showed that the first storm expanded more rapidly to lower latitudes, creating complex events in the ionosphere, while the second storm evolved more gradually and was more regular in nature. Other details and post factum analysis of this space weather event can also be found in work by Yermolaev et al. [2005].

[55] Real-time and quasi-real-time geomagnetic data available around the time of the storms proved that the ground-based observations can provide a detailed timeline for geomagnetic variability. At the same time, presentation of the global geomagnetic activity as maps would show the expansion of the global geomagnetic disturbances better.

[56] Ionospheric data, in contrast, provide many different types of event descriptions, among which maps are popular. An essential part of the maps should be to show the data locations and areas where embedded or statistical models predominate in the map construction, which is lacking in the presented maps. In comparison with the ground data, the solar and interplanetary observations cover a far larger spatial region, where three-dimensional maps of CME propagation are required and still remain a hope for the future.

[57] Forecasts of this complex space weather event demonstrated the importance of real-time data at all stages, from CME characteristics to the ground responses. Unfortunately, because of the complexity of the processes, space weather forecasts cannot follow the straightforward scheme of single solar cause-single ground response. Out of nine CME recorded, only two geoeffective ICME structures enveloped the Earth's magnetosphere, and the forecasts of their magnetic field direction caused problems even when all possible solar data were available. The geomagnetic forecasts (mostly) underestimated the severity of the first and second storms and overestimated the activity on 10-13 November, expected from later CMEs, although the real-time geomagnetic data undoubtedly helps to increase the reliability of the geomagnetic forecasts. The ionospheric forecasts, specifically if issued in the form of maps, were problematic because of the fast expansion of the disturbances to the lower latitudes and the nonuniformity of the ionosphere, coupled with the 
sparseness of data in some regions. Reliable statistical patterns of the storm time ionosphere over different regions might help to increase the accuracy of the ionospheric forecasts. Finally, the forecast of the characteristics of the ICME, such as amplitude of southward IMF component and its time of arrival, still remains the key question to be answered.

[58] Acknowledgments. EIT, LASCO, and MDI data have been used courtesy of the SOHO/EIT SOHO/LASCO and SOHO/MDI consortiums. We thank the ACE SWEPAM and MAG instrument teams and the ACE Science Centre for providing the ACE data. All authors would like to express their appreciation to the teams providing real-time data from the GOES satellites, INTERMAGNET observatories, and EPRI Sunburst GIC project. L.T. would like to acknowledge the support of the Canadian Space Agency. S.M.S. and N.J. are grateful for funding of their research by the German State Government of Mecklenburg-Vorpommern under grant V230-630-08-TIFA-334. A.Z. and R.v.d.L. are thankful for cofunding of their activity by ESA project (16913/03/NL/ $\mathrm{LvH})$ as part of the SWENET/Space Weather Pilot Project. A.W.P.T. would like to acknowledge the support of Scottish Power plc and ESA through the ESA Space Weather Pilot Project. All authors thank ESA for the timely organization of the splinter session at the first European Space Weather Week, where most of the parts of the paper were presented.

\section{References}

Bothmer, V., and R. Shwenn (1998), The structure and origin of magnetic clouds in the solar wind, Ann. Geophys., 16, 1-24.

Gopalswamy, N., L. Barbieri, E. W. Cliver, G. Lu, S. P. Plunkett, and R. M. Skoug (2005), Introduction to violent Sun-Earth connection events of October-November 2003, J. Geophys. Res., 110, A09S00, doi:10.1029/2005JA011268.

Hudson, H. S., and E. W. Cliver (2001), Observing coronal mass ejection without coronographs, J. Geophys. Res., 106, 25,19925,214 .

Jakowski, N., S. Heise, A. Wehrenpfennig, S. Schlueter, and R. Reimer (2002), GPS/GLONASS based TEC measurements as a contributor for space weather forecasting, J. Atmos. Sol. Terr. Phys., 64, 729-735.
Jakowski, N., V. Wilken, S. Schlueter, S. M. Stankov, and S. Heise (2005), Ionospheric space weather effects monitored by simultaneous ground and space based GNSS signals, J. Atmos. Sol. Terr. Phys., 67, 1074-1084.

Lundstedt, H. (2005), The Sun, space weather and GIC effects in Sweden, Adv. Space Res., 37, 1182-1191, doi:10.1016/j.asr.2005.10.023.

Marubashi, K. (1986), Structure of the interplanetary magnetic clouds and their solar origin, Adv. Space Res., 6(6), 335-338.

McAllister, A. H., S. F. Martin, N. U. Crooker, R. L. Lepping, R. J. Fitzenreiter (2001), A test of real-time prediction of magnetic cloud topology and geomagnetic storm occurrence from solar signatures, J. Geophys. Res., 106, 29,185-29,194.

Oler, C. (2004), Prediction performance of space weather forecast centers following the extreme events of October and November 2003, Space Weather, 2, S08001, doi:10.1029/2004SW000076.

Stanisławska, I., and Z. Zbyszyński (2001), Forecasting of the ionospheric quiet and disturbed $f_{\mathrm{o}} F_{2}$ values at single location, Radio Sci., 36(5), 1065-1071.

Stanisławska, I., G. Juchnikowski, and Z. Zbyszyński (2001), Generation of instantaneous maps of ionospheric characteristics, Radio Sci., 36(5), 1073-1081.

Thomson, A. W. P., A. J. McKay, E. Clarke, and S. J. Reay (2005), Surface electric fields and geomagnetically induced currents in the Scottish Power grid during the 30 October 2003 geomagnetic storm, Space Weather, 3, S11002, doi:10.1029/2005SW000156.

Trichtchenko, L., and D. H. Boteler (2004), Modeling GIC using geomagnetic indices and data, IEEE Trans. Plasma Sci., 32(4), $1459-1467$

Yermolaev, Y. I., et al. (2005), A year later: Solar, heliospheric and magnetospheric disturbances in November 2004, Geomagn. Aeron., 45(6), $681-719$.

Yurchyshyn, V. B., H. Wang, P. R. Goode, and Y. Deng (2001), Orientation of the magnetic fields in interplanetary flux ropes and solar filaments, Astrophys. J., 563, 381-388.

N. Jakowski and S. M. Stankov, Institute of Communications and Navigation, German Aerospace Centre, Kalkhorstweg 53, D-17235 Neustrelitz, Germany.

G. Juchnikowski and I. Stanisławska, Space Research Centre, Bartycka 18a str, 00-716 Warsaw, Poland.

G. Patterson and P. Wilkinson, IPS Radio and Space Services, 477 Pitt Street, PO Box 1386, Haymarket, NSW 1240, Australia.

A. W. P. Thomson, British Geological Survey, West Mains Road, Edinburgh EH9 3LA, UK.

L. Trichtchenko, Geomagnetic Laboratory, Natural Resources Canada, Ottawa, ON, Canada K1A 0Y3. (ltrichtc@nrcan.gc.ca)

R. van der Linden and A. Zhukov, SIDC, Royal Observatory, Av. Circulaire 3-Ringlaan 3, B-1180 Brussels, Belgium. 